\title{
Toll-like receptor engagement converts T-cell autoreactivity into overt autoimmune disease
}

\author{
Karl S Lang ${ }^{1,7}$, Mike Recher ${ }^{1,7}$, Tobias Junt ${ }^{1}$, Alexander A Navarini ${ }^{1}$, Nicola L Harris ${ }^{1}$, Stefan Freigang ${ }^{1}$, \\ Bernhard Odermatt ${ }^{1}$, Curdin Conrad ${ }^{2}$, Lars M Ittner ${ }^{3}$, Stefan Bauer ${ }^{4}$, Sanjiv A Luther ${ }^{5}$, Satoshi Uematsu ${ }^{6}$, \\ Shizuo Akira ${ }^{6}$, Hans Hengartner ${ }^{1} \&$ Rolf M Zinkernagel ${ }^{1}$
}

Autoimmune diabetes mellitus in humans is characterized by immunological destruction of pancreatic beta islet cells. We investigated the circumstances under which $C D 8^{+} T$ cells specific for pancreatic beta-islet antigens induce disease in mice expressing lymphocytic choriomeningitis virus (LCMV) glycoprotein (GP) as a transgene under the control of the rat insulin promoter. In contrast to infection with LCMV, immunization with LCMV-GP derived peptide did not induce autoimmune diabetes despite large numbers of autoreactive cytotoxic $T$ cells. Only subsequent treatment with Toll-like receptor ligands elicited overt autoimmune disease. This difference was critically regulated by the peripheral target organ itself, which upregulated class I major histocompatibility complex (MHC) in response to systemic Toll-like receptor-triggered interferon- $\alpha$ production. These data identify the 'inflammatory status' of the target organ as a separate and limiting factor determining the development of autoimmune disease.

Autoimmune diabetes in humans is characterized by immunological destruction of beta islet cells in the pancreas and subsequent hyperglycemia. T cells specific for beta islet cell antigens are likely to have a major role in the disease process ${ }^{1}$ and have been suggested to arise following exposure to viruses ${ }^{2-5}$ or infectious agents containing cross-reactive epitopes (molecular mimicry) ${ }^{6}$. Indeed, Coxsackie virus and human cytomegalovirus contain proteins that share amino acid sequences with human GAD65, which is strongly expressed in the pancreas ${ }^{7,8}$, and antigenic mimicry may explain the known association of human leukocyte antigen (HLA) haplotype and disease development ${ }^{5}$. However, several viruses suspected to be associated with diabetes do not contain any known cross-reactive epitopes 5 , and approximately 50\% of the European population is infected with human cytomegalovirus ${ }^{9}$ without being diabetic, although human cytomegalovirus has a crossreactive epitope with the beta islet antigen GAD65 (ref. 5). Autoreactive $\mathrm{T}$ cells have been detected in individuals who do not show overt disease ${ }^{10}$, suggesting that the onset of autoimmune disease is not determined solely by the presence of autoreactive $T$ cells.

To find an explanation for these discrepancies, we examined RIP-GP mice, in which LCMV-GP is expressed as a transgene under the control of the rat insulin promoter (RIP) ${ }^{11-14}$. These mice have been previously used to show that diabetes can be induced by autoreactive cytotoxic $\mathrm{T}$ cells (CTL) that are primed by LCMV infection ${ }^{11}$ or by injection of peptide-pulsed mature dendritic cells (DCs) ${ }^{13}$. Disease could not be induced by infection with LCMV-GP-recombinant vaccinia virus
(VV-G2), despite the presence of activated autoreactive $\mathrm{T}_{\text {cells }}{ }^{14}$. These different outcomes may have resulted from variation in the magnitude of the T-cell response induced. Alternatively, LCMV, or mature DCs, may provide nonspecific inflammatory signals in addition to activating GP-specific T-cell responses, which could influence the efficiency of $\mathrm{CD}^{+} \mathrm{T}$ cell-mediated killing ${ }^{12}$, for instance by increasing expression of MHC I on the target organ ${ }^{14}$ or by enhancing migration of $\mathrm{CD} 8^{+}$ $\mathrm{T}$ cells. Indeed, virus-induced production of chemokines is known to be important for T-cell migration into peripheral tissues and efficient antigen clearance ${ }^{15}$, and chemokine production in pancreatic islets has been shown to have a role in T-cell infiltration into this organ ${ }^{16}$. Tolllike receptor (TLR) signaling following recognition of distinct pathogen patterns can also influence the production of proinflammatory cytokines and chemokines by DCs and macrophages ${ }^{17-21}$. Eleven different TLRs have been identified so $\mathrm{far}^{22}$, and these recognize molecular patterns such as RNA (receptors TLR3, TLR7 and TLR8) ${ }^{23,24}$, DNA $\left(\right.$ TLR9) ${ }^{25}$ or lipopolysaccharide (TLR4) ${ }^{26}$. The pattern of cytokine production following TLR ligation depends on the type of TLR, which results in a specifically optimized pathogen-host balance and may occasionally result in autoimmune diseases ${ }^{27}$.

To analyze the role of such nonspecific immune stimuli in autoimmunity, we analyzed the induction of diabetes in RIP-GP mice following immunization with LCMV or with the immunodominant LCMV-GP-derived MHC class I peptide gp33 with or without additional TLR ligation.

\footnotetext{
${ }^{1}$ Institute of Experimental Immunology, University Hospital of Zurich, Schmelzbergstrasse 12, Zurich, Switzerland. ${ }^{2}$ Department of Dermatology, University Hospital of Zurich, Gloriastrasse 31, Zurich, Switzerland. ${ }^{3}$ Research Laboratory for Calcium Metabolism, Clinic Balgrist, University of Zurich, Forchstr. 340, Zurich, Switzerland. ${ }^{4}$ Institut für Medizinische Mikrobiologie, Immunologie und Hygiene, TU Munich, Trogerstr. 9, Munich, Germany. ${ }^{5}$ Department of Biochemistry, University of Lausanne, Chemin des Boveresses 155, Epalinges, Switzerland. ${ }^{6}$ Department of Host Defense, Osaka University, 3-1 Yamada-oka, Suita Osaka, Japan. ${ }^{7}$ These authors contributed equally to this work. Correspondence should be addressed to K.L. (karl.lang@usz.ch).
} 
a

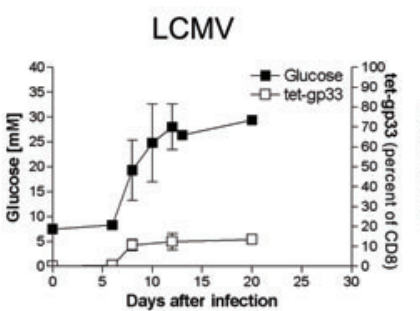

b

C $\quad \mathrm{CpG}+\mathrm{gp} 33$
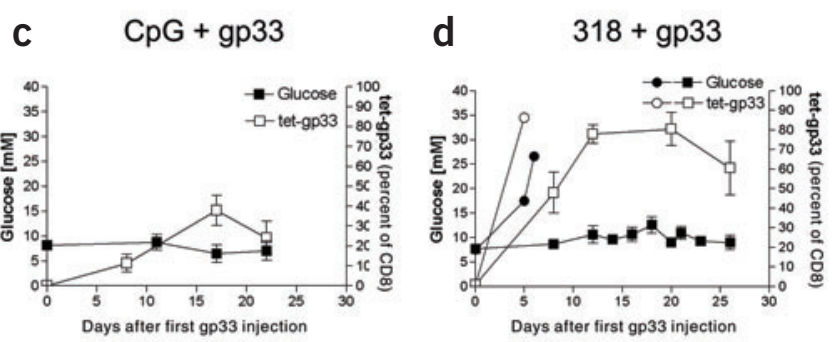

e $4 / 4$

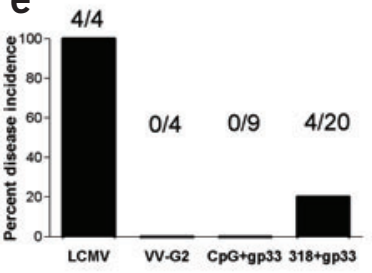

Figure 1 Expansion of $\mathrm{CD} 8^{+} \mathrm{T}$ cells specific for beta islet cell antigen alone does not necessarily induce diabetes. Filled square and filled circle, blood glucose concentrations. Open square, open circle, frequency of LCMV-GP ${ }_{33-41}$ (gp33) specific CD8+ T cells (tet-gp33+). (a) RIP-GP mice infected with 200 p.f.u. LCMV-WE intravenously $(n=3)$ (b) RIP-GP mice infected with $2 \times 10^{6}$ p.f.u. vaccinia virus recombinant for LCMV-GP intraperitoneally (VV-G2, $n=3$ ) (c) RIP-GP mice immunized with gp33 (1 mg in PBS) with CpG (ODN-1826, $50 \mu \mathrm{g}$ in phosphate-buffered saline) on days 0,2 and 5 . On day 9, mice were challenged with peptide alone $(n=3)$. (d) RIP-GP mice injected intravenously with $10^{7}$ splenocytes from LCMV-gp33/H-2Db-specific 318 TCR-transgenic mice and immunized with gp33 ( $1 \mathrm{mg}$ in PBS) on days 0,4 and $9(n=5)$. Filled square and open square, mice with normal blood glucose levels. Filled circle and open circle, mice developing diabetes. Eighty percent of mice showed normal blood glucose levels, and $20 \%$ developed diabetes. (e) Percentage of mice with diabetes at any time point following treatment protocols outlined in a-d.

\section{RESULTS}

\section{Activated autoreactive T cells without disease}

Mice expressing LCMV-GP as a transgene under the control of RIP were infected with 200 plaque-forming units (p.f.u.) of LCMV-WE (intravenous). Consistent with previous observations ${ }^{11,14}$, infection resulted in expansion of LCMV-GP ${ }_{33-41}$-specific $\mathrm{CD} 8^{+} \mathrm{T}$ cells identified by tetramer binding (tet-gp $33^{+}$cells, Fig. 1a), and all infected mice developed hyperglycemia by day 8-10 (Fig. 1a,e). Infection with the vaccinia virus expressing the LCMV-GP recombinant VV-G2 led to reduced expansion of tetgp $33^{+}$cells (Fig. 1b), and no diabetes occurred (Fig. 1b,e). To directly investigate the role of CTL frequency in the induction of autoimmune disease, we immunized RIP-GP mice with the LCMV-GP-derived peptide gp33 together with CpG (ODN-1826) to deliver optimal costimulation. This immunization induced massive expansion of gp33-specific $\mathrm{CD} 8^{+} \mathrm{T}$ cells such that the frequency of these cells reached $40 \%$ of total $\mathrm{CD}^{+} \mathrm{T}$ cells (Fig. 1c). Nevertheless, all mice showed normal blood glucose concentrations, indicating the absence of disease (Fig. 1c,e). To further increase autoreactive T-cell frequencies, we adoptively transferred gp33-specific TCR-transgenic splenocytes (318 CD8 ${ }^{+} \mathrm{T}$ cells) into RIP-GP mice and immunized recipient mice with gp33 peptide alone. This protocol resulted in an expansion of the gp33-specific T cells up to a frequency of $80 \%$ of total $\mathrm{CD}^{+} \mathrm{T}$ cells (corresponding to $30 \%$ of total lymphocytes) even in the absence of costimulatory CpG (Fig. 1d). Still, only $20 \%$ of these mice developed hyperglycemia (Fig. 1d,e).

We reasoned that the absence of diabetes in RIP-GP mice showing high frequencies of gp33-specific $\mathrm{CD} 8^{+} \mathrm{T}$ cells could be explained by inadequate CTL effector function following peptide priming. We therefore examined the functional status of the gp33-specific $318 \mathrm{CD}^{+} \mathrm{T}$ cells following peptide immunization. Analysis of surface marker expression showed a CD44 high CD62L ${ }^{\text {low }}$ phenotype suggestive of activation (Fig. 2a). These cells also expressed high levels of the markers Ly $6 c+g$ and IL-2 receptor $\beta$-chain (CD122, Fig. 2a), generally expressed by activated T cells. Peptide-stimulated TCR transgenic $\mathrm{CD}^{+} \mathrm{T}$ cells produced interferon- $\gamma$ (IFN- $\gamma$ ) following in vitro restimulation (Fig. $2 \mathbf{b}$ ) and lysed peptide-pulsed target cells in ex vivo primary and secondary ${ }^{51} \mathrm{Cr}$ release cytotoxicity assays (Fig. 2c). Furthermore, peptide-immunized mice were able to eliminate gp33-labeled target splenocytes within $4 \mathrm{~h}$ in vivo, suggesting full lytic function in vivo of the gp33-specific 318 CTLs present in these mice (Fig. 2d).

Mice immunized with gp33 plus CpG (ODN-1826) without previous transfer of transgenic $318 \mathrm{CD}^{+} \mathrm{T}$ cells mounted a similar functional CTL response as shown by the ability of these mice to clear gp33-pulsed splenocytes in vivo within $4 \mathrm{~h}$ and an LCMV infection within $4 \mathrm{~d}$ (Fig. 2e,f).

\section{Pancreatic T-cell infiltrations without disease}

We next examined whether the functional beta islet cell-specific CTLs present in gp33 immunized mice did not induce diabetes because of the lack of migration into the pancreas. To address this issue, we crossed the RIP-GP mice with mice expressing the chemokine CXCL13/BLC under control of the rat insulin promoter (RIP-BLC), which have been previously described to display lymphoid infiltrations within the endocrine pancreas ${ }^{28}$. Mice doubly transgenic for RIP-GP and RIP-BLC were transfused with transgenic gp33-specific splenocytes (318), and recipient mice were immunized with gp33. This resulted in a rapid expansion of gp33-specific $\mathrm{CD}^{+} \mathrm{T}$ cells, such that their frequency approached $50 \%$ of total lymphocytes in blood (Fig. 3a). Infiltration of these peptide-activated CTLs into the pancreatic islets was shown by immunohistochemical analysis of the Vo2 TCR expressed by 318 CD ${ }^{+}$T cells (Fig. 3c), yet none of the mice developed diabetes (Fig. 3a). This indicated that the presence of functional CTLs within the pancreas was not sufficient to induce diabetes. In contrast, infection of these mice with LCMV resulted in hyperglycemia (Fig. 3b), suggesting that some factor (other than CTL activation and migration into the pancreas) associated with viral infection had mediated induction of disease.

\section{MHC class I upregulation by LCMV versus pepitide}

We reasoned that the ability of LCMV infection to induce diabetes could be explained by virus-induced cytokine production rendering pancreatic beta islet cells susceptible to CTL-mediated destruction. Four days after LCMV infection, we detected strongly elevated serum IFN- $\alpha$ (Fig. 4a), a cytokine known to induce the upregulation of MHC class $\mathrm{I}^{29}$. Indeed, immunohistochemical analysis of beta islet cells infected with LCMV showed enhanced expression of MHC class I in LCMV-infected mice 
(Fig. 4b), consistent with previous reports ${ }^{12,14}$. In contrast, immunization with gp33 enhanced neither systemic IFN- $\alpha$ production nor MHC class I expression in pancreatic beta islet cells (Fig. 4a,b). Rag1 ${ }^{-/-}$mice (lacking $\mathrm{T}$ and $\mathrm{B}$ cells) showed a smaller enhancement of MHC I expression after LCMV infection when compared with naive and LCMV-infected C57BL/6 mice (Fig. 4c). Additional functional depletion of the interferon (IFN) system in mice lacking type I $\left(\right.$ Ifnar $\left.^{-/}\right)$, type II $\left(\right.$Ifngr $\left.^{-/}\right)$or both (Ifnagr ${ }^{-/}$Rag1 $^{-/-}$) IFN receptors abrogated MHC class I expression after LCMV infection. Notably, virus replication within beta islet cells was detected only in mice lacking IFN type I receptors (Fig. 4c).

\section{TLRs regulate MHC class I expression on beta cells}

IFN- $\alpha$ is produced after ligation of TLRs with virus-related products (e.g., RNA) specifically engaging TLR3 and TLR7 (refs. 24,30,31). We therefore analyzed the involvement of TLR stimulation in the production of systemic IFN- $\alpha$ and upregulation of MHC class I on beta islet cells. Administration of the virus-related double-stranded RNA repeat poly $(\mathrm{I}: \mathrm{C})^{23}$ to naive $\mathrm{C} 57 \mathrm{BL} / 6$ mice resulted in substantial production of IFN- $\alpha$ and upregulation of MHC class I expression on beta islet cells (Fig. 5a,c and Supplementary Fig. 1 online). This effect could be mimicked by comparable amounts of recombinant IFN- $\alpha$ (Fig. 5b). As observed for LCMV infection, poly(I:C)-induced upregulation of MHC I expression on beta islet cells required functional IFN type I receptor signaling, as shown using Ifnar ${ }^{-/-}$mice (Fig. 5d). Poly(I:C)-triggered MHC I upregulation was mediated by TLR3 stimulation, being absent in $\mathrm{Tlr} 3^{-/}$mice (Fig. 5d). Treatment of C57BL/6 mice with the low molecular weight immune modifier R-848 (a ligand for TLR7) ${ }^{32}$ similarly enhanced serum IFN- $\alpha$ production and pancreatic MHC class I expression (Fig. 5a,c and Supplementary Fig. 1 online). We found that IFN- $\alpha$ was essential for R-848-mediated upregulation of $\mathrm{MHC}$ class I expression, as this response was blocked by a monoclonal antibody specific for IFN- $\alpha$ (RMMA-1, Fig. 5e). In addition, mice deficient in type I IFN receptor (Ifnar ${ }^{-/}$) were not able to upregulate MHC I following R-848 treatment (Fig. 5e).

Figure 2 Peptide immunization induces highly activated CTLs. (a) Expression of CD44, CD62L, Ly6c+g and CD122 as determined by FACS. Numbers represent percentage of cells Ly $6 c+g^{\text {high }} \mathrm{CD} 122^{\text {high }}$ (bottom) or CD44 ${ }^{\text {high }} \mathrm{CD} 62 \mathrm{~L}^{\text {low }}$ (top). All plots representative of data obtained from four animals. (b) Intracellular production of IFN- $\gamma$ by splenocytes after in vitro stimulation with or without gp33. Numbers represent percentage of CD8 ${ }^{+}$ T cells staining positively for IFN- $\gamma$. Forty-five percent of total $\mathrm{CD}^{+} \mathrm{T}$ cells were gp33-specific; therefore nearly all tetramer-positive $T$ cells produced IFN- $\gamma$. Both plots are representative of data obtained from four animals. (c) In vitro lytic activity as analyzed by primary and secondary ${ }^{51} \mathrm{Cr}$ release assay. (d) In vivo killing activity as analyzed by an in vivo killer assay. Black line, splenocytes injected into gp33-immunized mice. Gray area, splenocytes injected into naive RIP-GP mice. Plots are representative of data obtained from three animals and show approximately $85 \%$ lysis of gp33-loaded cells in immunized recipients. (a-d) $10^{7}$ splenocytes from LCMV-gp33/H-2D $\mathrm{D}^{\mathrm{b}}$ specific TCR-transgenic 318 mice were transferred (intravenously) into naive RIP-GP mice later immunized with gp33 (1 mg in phosphate-buffered saline) on days 0, 4 and 9. Splenocytes were removed for analysis between day 11 and 14 . (e) In vivo killing activity of mice immunized with gp33 and CpG. FACS plots are representative of data obtained from three animals and show approximately $70 \%$ lysis of gp33-loaded cells in immunized recipients. (f) Spleen virus titers of individual C57BL/6 mice as analyzed by plaque assay. Mice were infected with 200 p.f.u. LCMV at day 30 or 80 after immunization and titers analyzed four days later. All mice in $\mathbf{e}$ and $\mathbf{f}$ were immunized with $1 \mathrm{mg}$ gp33 together with CpG (ODN-1826, $50 \mu \mathrm{g})$ as described in Fig. 1c, or remained unmanipulated (Naive). For all panels: $\varnothing$, without peptide. gp33, with gp33. Naive, tet-gp33- $\mathrm{CD}^{+} \mathrm{T}$ naive cells. Primed, peptide-primed tet-gp $33^{+} \mathrm{CD} 8^{+} \mathrm{T}$ cells.

Upregulation of pancreatic MHC class I expression following $\mathrm{R}-848$ treatment was mediated by specific TLR signaling as shown in $T l r 7^{-/-}$mice (Fig. 5e). R-848 induced upregulation of MHC class I expression required the presence of the common TLR adaptor protein MyD88 expressed on bone marrow-derived cells (Fig. 5e). This strongly suggested that bone marrow derived cells are responsible for the observed MHC I upregulation, whereas pancreatic islet cells themselves do not respond to R-848. Triggering of TLR4 by lipopolysaccharide, or TLR9 by CpG (ODN-1826) did not result in upregulated MHC class I expression on beta islet cells (Fig. 5c and Supplementary Fig. 1 online), consistent with the inability of the employed dose of $\mathrm{CpG}$ to enhance systemic IFN- $\alpha$ levels (Fig. 5a) ${ }^{33}$. Thus, in our experiments, the quantities of IFN- $\alpha$ required for pancreatic islet cell MHC class I upregulation could only be provided through LCMV infection or by stimulation of TLR-3 and TLR-7.

To determine the source of IFN- $\alpha$ following poly(I:C) treatment, we analyzed sequential sections of spleens taken from mice that had received poly(I:C) $3 \mathrm{~h}$ earlier. We detected the majority of IFN- $\alpha$ in cell clusters in the marginal zone (Supplementary Fig. 2 online), consistent with earlier reports ${ }^{20,34}$.

\section{TLRS convert autoreactivity into overt disease}

We showed that LCMV infection leads to the upregulation of MHC class I on beta islet cells and that this effect could be mimicked by injection of recombinant IFN- $\alpha$, the TLR3 ligand poly(I:C), or the TLR7

a

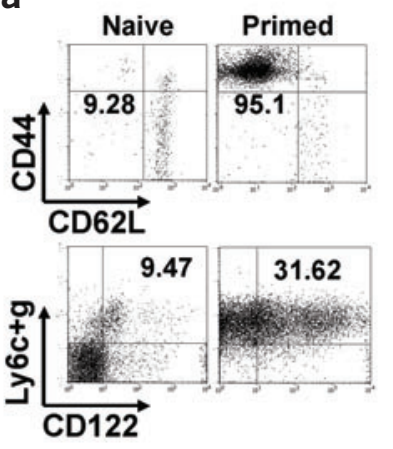

C

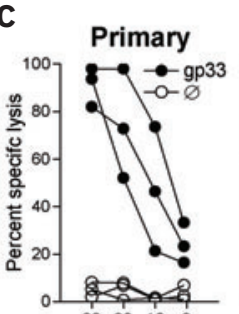

$90 \quad 30 \quad 10 \quad 3$

Ratio (effector: target)

f

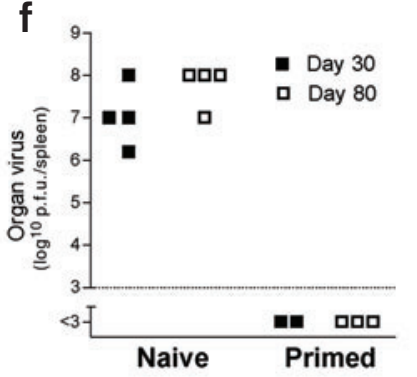

b

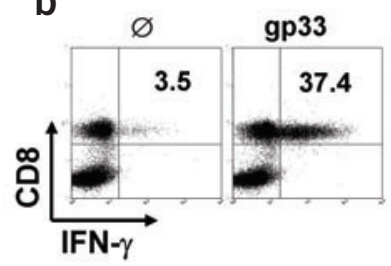

d

$318+\operatorname{gp} 33(4 \mathrm{~h})$

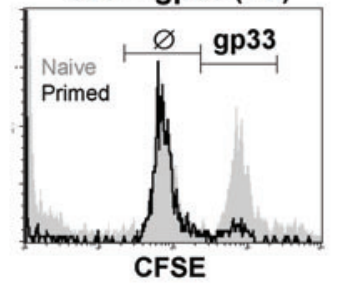

e

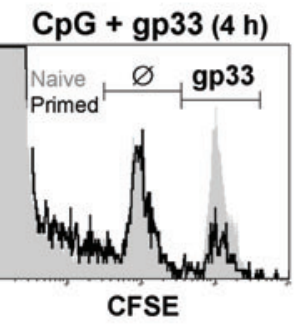


a

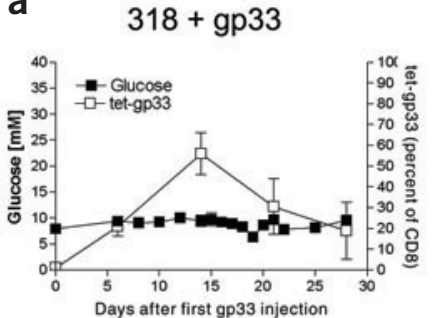

C
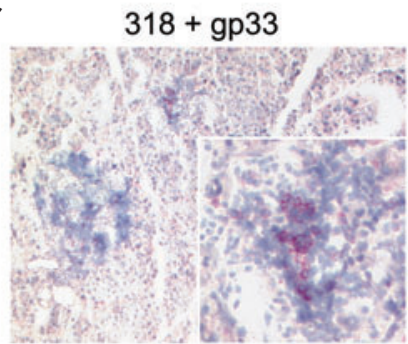

b

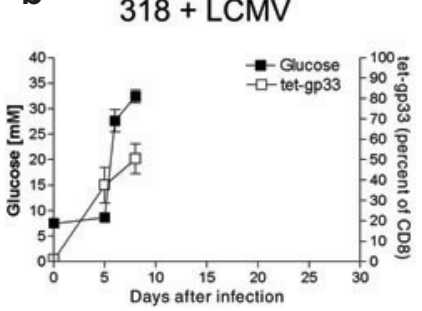

Figure 3 Pancreatic infiltration by activated beta islet-specific CD8 ${ }^{+} \mathrm{T}$ cells is not sufficient to induce diabetes. $10^{7}$ splenocytes from LCMV-gp33/H-2 $\mathrm{D}^{\mathrm{b}}$ specific $V \alpha 2^{+}$TCR-transgenic 318 mice were transferred into mice doubly transgenic for RIP-GP and RIP-BLC. Mice were then (a) immunized with gp33 ( $1 \mathrm{mg})$ on days 0,4 and $9(n=4)$ or (b) infected with 200 p.f.u. LCMV on day $0(n=3)$. Blood glucose concentration (filled square) and percent of gp33-tetramer positive cells (open square) (as percentage of total $C D 8^{+} \mathrm{T}$ cells) were monitored at the indicated time points. (c) Twenty days following the last peptide immunization, naive or gp33-immunized mice were killed and the pancreas examined for the presence of $\mathrm{V} \alpha 2^{+} \mathrm{CD} 8+\mathrm{T}$ cells using immunohistochemistry $(n=2)$.

nized mice did not become diabetic after LCMV infection (Fig. $\mathbf{5 g}$ ). This was possibly due to rapid viral clearance by primed $\mathrm{CD} 8^{+}$ $\mathrm{T}$ cells and thus reduced IFN- $\alpha$ production in immunized mice (Fig. 5g). To further strengthen our hypothesis that TLR signals are required in addition to autoreactive $\mathrm{T}$ cells to induce overt autoimmune disease, we investigated RIP-GP mice deficient in the common TLR adaptor molecule MyD88. Bone marrow chimeras were generated by injection of $M y d 88^{-/-}$bone marrow into lethally irradiated RIP-GP or C57BL/6 mice. After LCMV immunization, C57BL/6 bone marrow chimeras ( $M y d 88^{-1-}$ bone marrow into C57BL/6) had reduced pancreatic MHC I expression on day 8 after infection (Fig. 6a), and no autoimmune disease was observed in RIP-GP chimeras

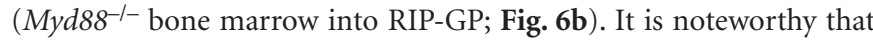
in these mice, autoreactive $\mathrm{CD} 8^{+} \mathrm{T}$ cells showed a delayed expansion after LCMV infection (Fig. 6c), indicating a role for TLR signals in both innate and adaptive immune responses. But tet-gp $33^{+} \mathrm{CD} 8^{+} \mathrm{T}$ cells that were present were functional, as assessed by intracellular IFN- $\gamma$ staining and FACS analysis (Fig. 6e) as well as in a direct ex vivo cytotoxicity

ligand R-848. This prompted us to investigate whether autoimmune diabetes could be elicited by administration of recombinant IFN- $\alpha$ or 을 by ligation of TLR3 or TLR7 in RIP-GP mice that were preimmunized with gp33 in the absence of virus infection. Indeed, administration of IFN- $\alpha$, poly(I:C) or R-848 to these peptide-immunized mice resulted in a rapid and sometimes transient development of hyperglycemia (Fig. 5f). The ability of the TLR ligands to induce disease thus correlated strictly with their capacity to induce systemic IFN- $\alpha$ and to upregulate MHC class I on pancreatic islet cells (Fig. 5a-c and Supplementary Fig. 1 online). In contrast to naive mice, gp33-immu-

a

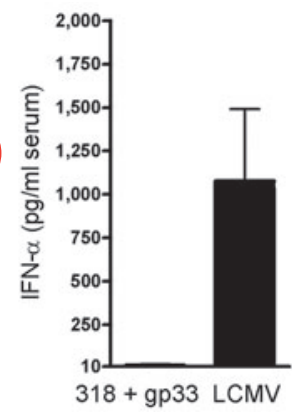

b

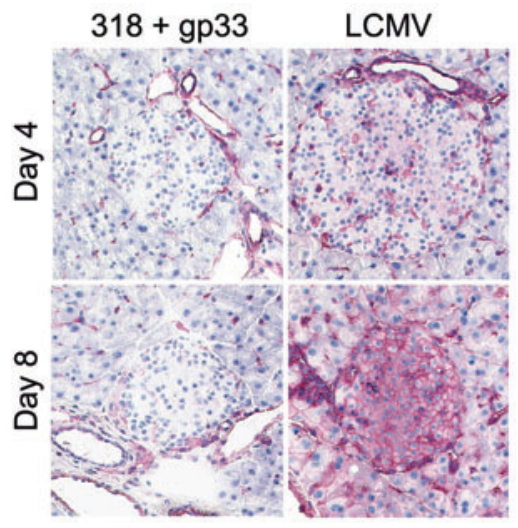

C

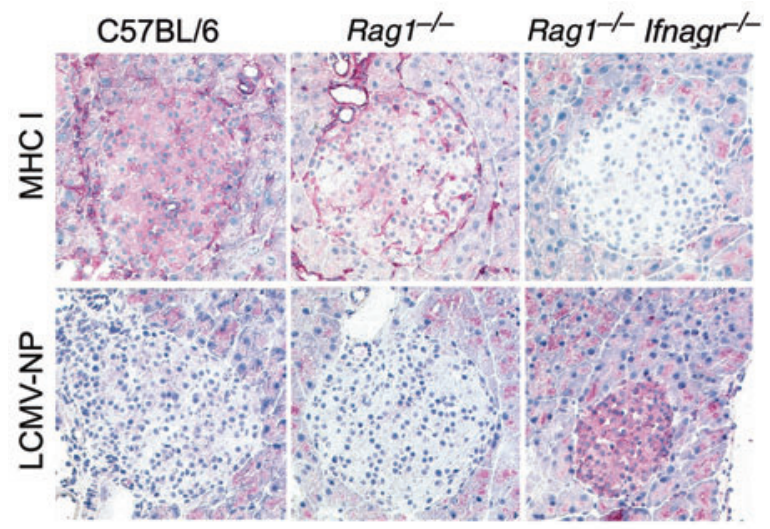

Figure 4 LCMV infection, but not peptide immunization, results in MHC class I upregulation on beta islet cells. (a,b) $10^{7}$ splenocytes from LCMVgp33/H-2D ${ }^{b}$-specific TCR-transgenic 318 mice were transferred to C57BL/6 mice which were then immunized with $1 \mathrm{mg}$ gp33 on day 0 and 2 or infected with 200 p.f.u. LCMV on day 0. (a) Serum IFN- $\alpha$ concentration was determined by ELISA on day 4 . $(n=5-6)$

(b) Pancreatic tissue was examined by immunohistochemistry for $\mathrm{MHC}$ class I expression on day 4 and day $8(n=3)$. (c) C57BL/6, Rag1 $^{-1-}$ (on C57BL/6 background) Ifnagr ${ }^{-1}$ Rag1 $^{-1-}$ (on a mixed background, C57BL/6 × 129Sv), 129Sv, Ifnar ${ }^{-1}$, and Ifngr ${ }^{-1}$ mice (on 129 Sv background) were infected with 200 p.f.u. LCMV. Eight days later, pancreatic tissue was stained with a general antibody specific for $\mathrm{MHC}$ class I (M1/42, staining H2-b and H2-d) and LCMV nucleoprotein as indicated (LCMV-NP, $n=2-3$ ).

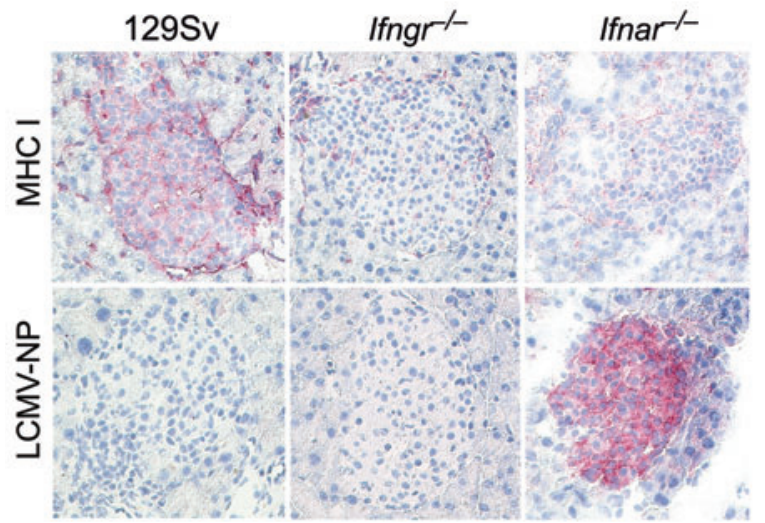


a

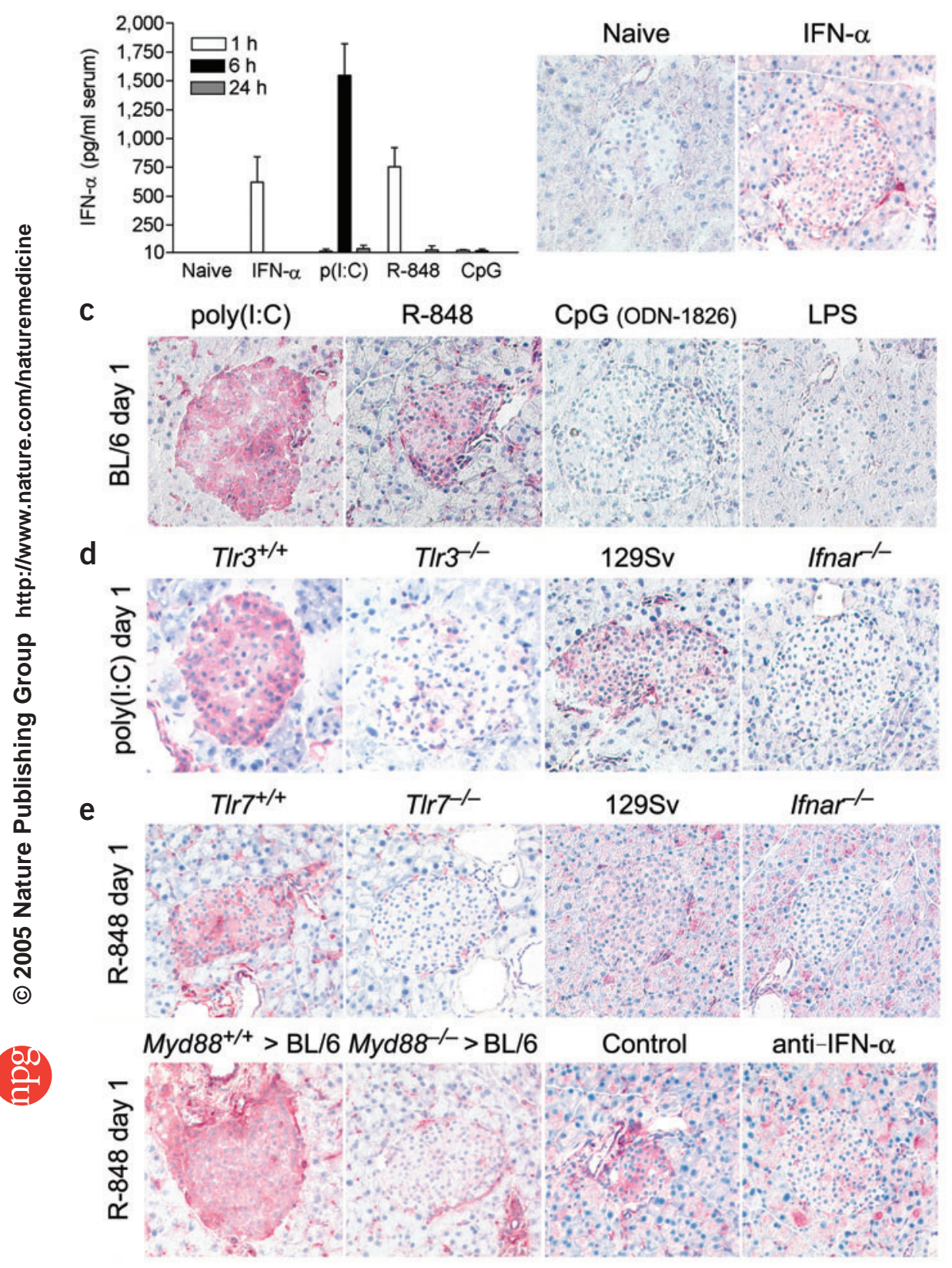

Figure 5 Toll-like receptor stimulation induces $\mathrm{MHC}$ class I upregulation on beta islet cells. (a) C57BL/6 mice were treated intravenously with recombinant IFN- $\alpha$ ( $10^{4}$ units), poly(I:C) (200 $\mu \mathrm{g}), \mathrm{R}-848$ (20 $\mu \mathrm{g})$ or CpG (ODN-1826, $50 \mu \mathrm{g}$ ) and the serum concentration of IFN- $\alpha$ was determined by ELISA 1, 6 and $24 \mathrm{~h}$ later ( $n=3$; only the $1 \mathrm{~h}$ value is shown for naive and IFN- $\alpha$ treatment). (b) $\mathrm{C} 57 \mathrm{BL} / 6$ mice were treated with IFN- $\alpha$ ( $10^{4}$ units) and endocrine pancreatic tissue analyzed for MHC class I expression 24 $\mathrm{h}$ later $(n=3)$ with a general anti-MHC class I antibody (M1/42, staining $\mathrm{H} 2-\mathrm{b}$ and $\mathrm{H} 2-\mathrm{d}$ ). (c) $\mathrm{C} 57 \mathrm{BL} / 6$ mice were treated with poly (I:C) (200 $\mu \mathrm{g}), \mathrm{R}-848(20 \mu \mathrm{g}), \mathrm{CpG}$ (ODN-1826, 50 $\mu \mathrm{g})$ or lipopolysaccharide $(50 \mu \mathrm{g})$ on day 0 . At day 1 , endocrine pancreatic tissue was analyzed for MHC class I expression $(n=3)$. Analysis for day 3 and 6 is shown in Supplementary Fig. 1 online. (d) $T / r 3^{-1-}$ mice and control BALB/c mice were treated with $500 \mu \mathrm{g}$ poly(I:C). Ifnar ${ }^{-1}$ and wild-type control mice (129Sv) were treated with $200 \mu \mathrm{g}$ poly(I:C). Twenty-four hours later, the pancreas was removed and examined for expression of islet-cell MHC class I. (e) TIr $7^{-1-}$ mice, Ifnar ${ }^{-1}$ mice, MyD88-deficient bone marrow chimeras ( $M y d 88^{-1-}$ bone marrow cells into C57BL/6 recipients), C57BL/6 mice treated with a monoclonal neutralizing antibody specific for IFN- $\alpha$ (RMMA-1, $30 \mu \mathrm{g} /$ mouse on day 0 ) and corresponding control mice were treated with $20 \mu \mathrm{g}$ R-848. Twenty-four hours later pancreatic tissue was analyzed for MHC class I expression. $(\mathbf{f}, \mathbf{g})$ RIP-GP mice were immunized with gp33, with or without transfer of $10^{7}$ splenocytes derived from LCMV-gp33/H-2D $\mathrm{D}_{\text {-specific TCR- }}$ transgenic 318 mice. Mice with $>20 \%$ of gp33specific $\mathrm{CD}^{+} \mathrm{T}$ cells in the peripheral blood were further analyzed for blood glucose concentration (filled circle). Blood glucose concentration of C57BL/6 or RIP-GP mice that had not received gp33 immunization (open circle), or did not receive additional treatment (open square), served as controls. Mice were treated intravenously with $10^{4}$ units IFN- $\alpha(n=5), 200 \mu \mathrm{g}$ of

poly (I:C) (total $n=5$ ) or $20 \mu \mathrm{g}$ of R-848 $(n=4)$ at day 5 and 11 (day 3 for IFN- $\alpha$ ) following the last peptide immunization. (g) At day 10-20 after the last peptide immunization, mice were challenged with 200 p.f.u. of LCMV (filled circle). LCMV treated mice were analyzed for IFN- $\alpha$ on day $4(n=3)$. Naive RIP-GP mice served as controls (open circle).
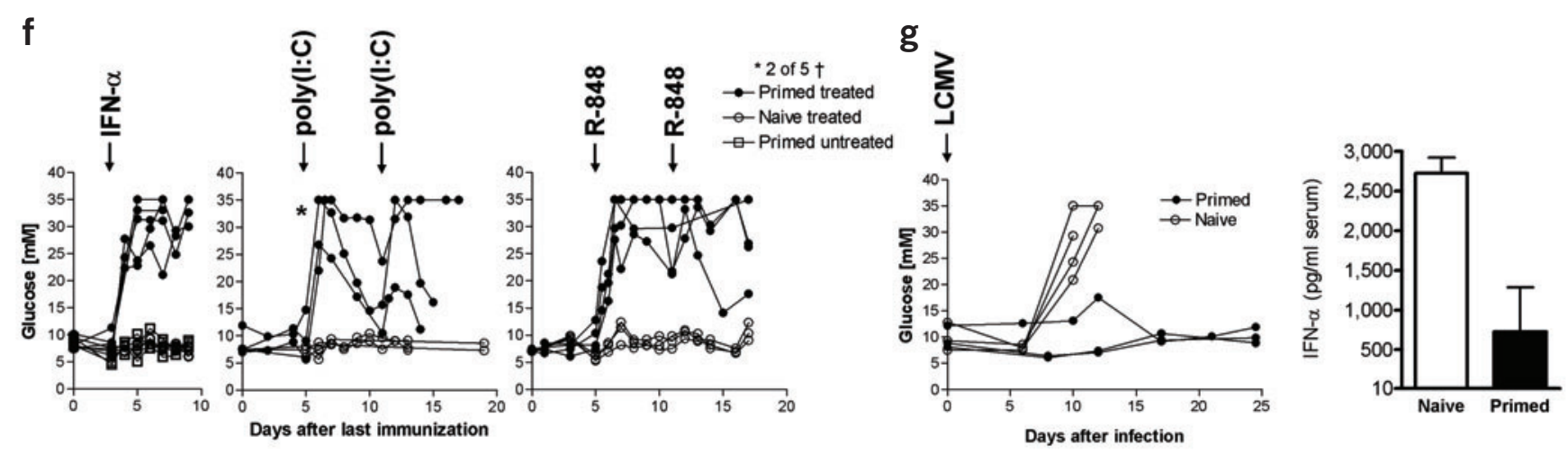


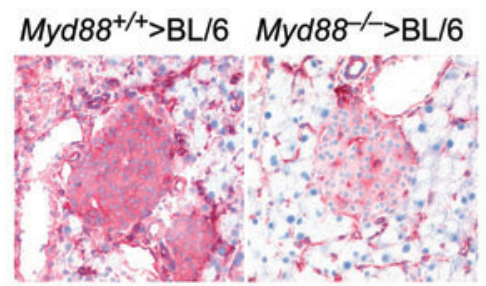

b

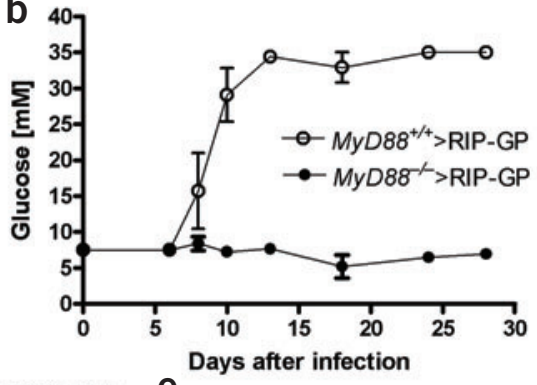

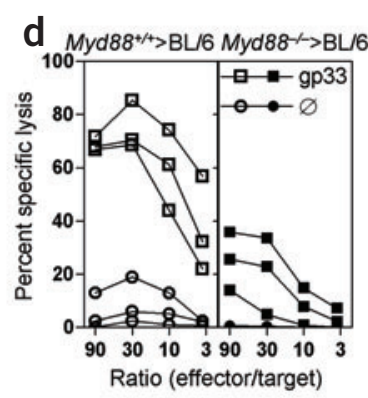

e
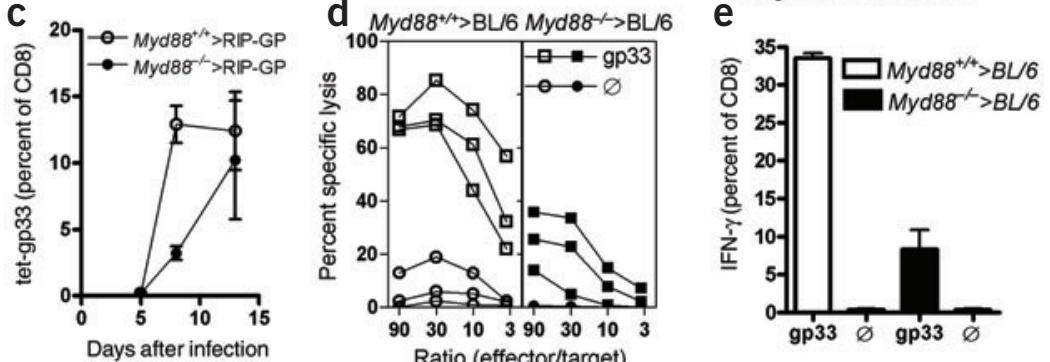

f

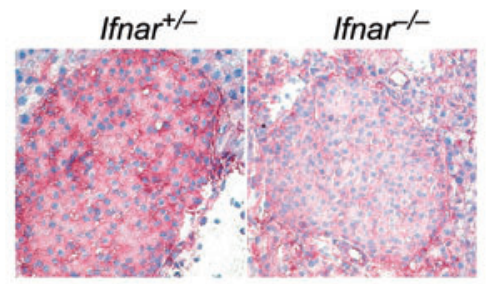

h

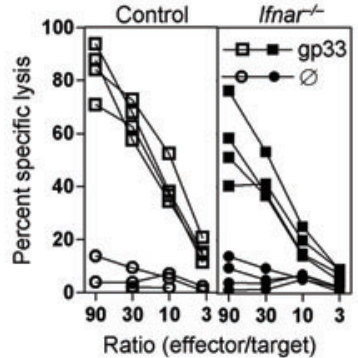

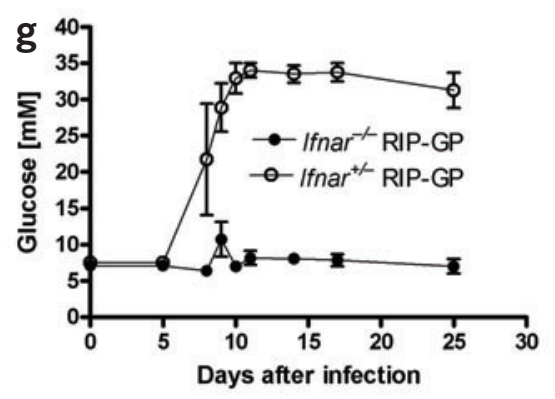

Figure 6 Prevention of LCMV-induced diabetes by lack of MyD88 or IFN type I receptor.

(a-e) $M y d 88^{-1-}$ or $M y d 88^{+/+}$bone marrow cells were adoptively transferred into irradiated C57BL/6 or RIP-GP mice. Myd88 ${ }^{-1}>$ C57BL/6 and $\mathrm{Myd} 88^{+/+}>\mathrm{C} 57 \mathrm{BL} / 6$ mice were infected with 200 p.f.u. LCMV (7 weeks later). (a) On day 8 after infection, MHC I expression on pancreatic islet cells was assessed by staining with an general antibody specific for MHC class I (M1/42, staining $\mathrm{H} 2-\mathrm{b}$ and $\mathrm{H} 2-\mathrm{d}$ ). $\mathrm{CD}^{+} \mathrm{T}$ cells of these mice were analyzed on the same day for direct ex vivo cytotoxicity (d) and for IFN- $\gamma$ production following a 6-h peptide restimulation in vitro

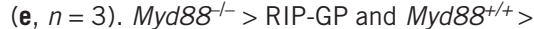
RIP-GP mice were infected with LCMV and blood sugar levels (b) and numbers of tet-gp33+ cells in the blood (c) were monitored $(n=4)$. ( $\mathbf{f}-\mathbf{h})$ IFN$\alpha$-deficient mice were crossed with RIP-GP mice. IFN- $\alpha$-deficient mice and control littermates (F3 generation 129Sv $\times$ C57BL/6) were infected with LCMV. On day 8 after infection, splenocytes were analyzed for direct ex vivo cytotoxicity (h). In RIP-GP-negative littermates, MHC class I expression on pancreatic islet cells was assessed by immunohistology on day 8 (f). Ifnar ${ }^{-/}$RIP-GP mice and Ifnar ${ }^{+/-}$RIP-GP mice were infected with LCMV and blood glucose was monitored (g, $n=4)$.

disease in mice expressing LCMV-GP under control of the rat insulin promoter. It has previously been shown that autoreactive $\mathrm{CD}^{+} \mathrm{T}$ cells without the chemokine CXCR3 cannot induce diabetes because of inefficient homing ${ }^{16}$. In our experiments using double transgenic RIP-GP $\times$ RIP-BLC mice, we found that GPspecific CTLs can be located within pancreatic endocrine tissue yet still not cause disease as long as the beta islet cells express only low levels of MHC I. Thus, although an initial clonal burst of autoreactive $\mathrm{CD} 8^{+} \mathrm{T}$ cells and their recruitment into the autoantigen-presenting peripheral organ are prerequisites for disease assay (Fig. 6d). Chimeras lacking MyD88 ( $M y d 88^{-/-}$bone marrow into C57BL/6) were able to initially suppress virus replication in a similar manner that observed in control chimeras (C57BL/6-negative bone marrow into C57BL/6).

\section{IFN type I is essential for virus-induced diabetes}

To directly examine the requirement for type I IFNs in mediating MHC class I upregulation on pancreatic islet cells and the subsequent induction of autoimmune disease, we crossed RIP-GP mice to Ifnar ${ }^{-1}$ mice and then infected these mice with LCMV. IFN type I receptor-competent RIP-GP mice developed diabetes with normal kinetics, whereas type I IFN receptor-deficient littermates remained healthy (Fig. 6g). Consistent with published evidence ${ }^{35}$, we observed normal $\mathrm{CD}^{+} \mathrm{T}$-cell priming in the Ifnar ${ }^{-1}$ mice, as assessed in a direct ex vivo cytotoxicity assay (Fig. 6h). MHC class I expression within pancreatic islet cells was reduced in mice lacking type I IFN signals (Fig. 6f).

\section{DISCUSSION}

We have shown that the presence of activated and functional gp33specific $\mathrm{CD}^{+} \mathrm{T}$ cells was not sufficient for induction of autoimmune immune disease

In our experiments, disease onset and progression was further determined by the 'inflammatory status' of the target organ, as indicated by MHC class I expression by beta islet cells. This upregulation of MHC class I expression on beta islet cells was dependent on functional IFN type I receptors and seemed to be critically regulated by production of systemic IFN- $\alpha$. It is likely that activation of IFN type I receptors by IFN- $\beta$ can also upregulate MHC I expression. IFN- $\beta$ is also produced during LCMV infection ${ }^{36}$, and it is possible that it contributes to diabetes in the LCMV system. This would be consistent with the finding that neutralizing IFN- $\alpha$ antibody treatment substantially delayed the onset of disease but did not prevent diabetes completely (K.L. and M.R., unpublished data), whereas a blockade of IFN type I receptor completely prevented diabetes.

Notably, cells within the pancreatic islets were more susceptible to upregulation of MHC class I expression than exocrine pancreatic tissue, consistent with previous observations ${ }^{12,14}$. We are currently evaluating whether this mechanism holds true for other endocrine cells; it may partially explain why autoimmune diseases often target induction, other factors exert additional control over the onset of auto- 
endocrine organs. There has been no previous characterization of the inflammatory status of beta islet cells during health or disease; however, treating hepatitis $\mathrm{C}$ virus-infected human individuals with IFN- $\alpha$ occasionally led to diabetes if beta cell-specific antibodies were present in the serum ${ }^{37}$. Mechanisms underlying this antibodymediated destruction of beta islets remain to be established.

Poly(I:C) was able to prevent diabetes in NOD (non-obese diabetic) mice ${ }^{38}$, but it was shown in diabetes-prone bio-breeding (DP-BB) rats that poly(I:C) can accelerate $\mathrm{e}^{39,40}$ or prevent diabetes, ๑ depending on the dosage used ${ }^{41}$. These contradictory observations may be partially explained by differences in doses and different kinetics of poly(I:C). Chronic application of TLR ligands is known to tolerize subsequent TLR triggering ${ }^{42,43}$. In addition, different doses of TLR ligands might influence the activation status of responding or anergized autoreactive $\mathrm{CD}^{+} \mathrm{T}$ cells differently.

Our manuscript shows how innate immune activation influences the adaptive immune response. Blocking TLR signaling

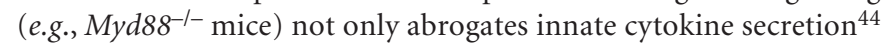
but also delays the priming of specific $\mathrm{T}$ cells. Specific $\mathrm{T}$ cells may themselves augment 'inflammatory' signals by production of cytokines (e.g., IFN- $\gamma$ ), which in our model may also contribute to upregulation of pancreatic MHC class I expression at late time points. In addition to TLR signaling, MyD88 is involved in signaling through the IL-1 and IL-18 receptors ${ }^{45,46}$. Notably, IFN responses induced by virus infection are enhanced in the presence of IL-18 (ref. 47). We therefore cannot exclude a role for these cytokines in the delayed diabetes induction found in our Myd88 ${ }^{-/-}$experiments.

In conclusion, our experiments using the LCMV RIP-GP model have shown that highly activated $C D 8^{+}$T cells can 'peacefully coexist' with pancreatic beta islet cells expressing the relevant autoantigen. Engagement of TLRs was required to break this coexistence and converted potential autoreactivity into overt autoimmune disease. This may indicate an important role for systemic virus infections in the disease-triggering phase of autoimmune diabetes in humans. In particular, a role for TLR signaling is consistent with the observation that overt diabetes often coincides with viral infections in human patients ${ }^{5}$. Alternatively, local inflammation within the pancreas may cause upregulation of MHC class I and promote autoimmunity. Taken together, our results suggest that pathogenesis of clinically relevant autoimmune diseases can be viewed as a two step process, requiring both the generation of autoreactive $T$ cells and TLR-mediated inflammation that renders the target organ susceptible to immune attack.

\section{METHODS}

Mice and viruses. LCMV strain WE was originally obtained from F. LehmannGrube (Heinrich Pette Institute) and was propagated in L929 cells. Virus titers were measured using a focus-forming assay as described ${ }^{48}$. Mice were infected with 200 p.f.u. LCMV-WE or with $2 \times 10^{6}$ p.f.u. recombinant vaccinia virus expressing the LCMV-GP (VV-G2). Mice transgenic for a T-cell receptor recognizing LCMV-GP ${ }_{33-41}$ (LCMV-gp33/H-2D ${ }^{\mathrm{b}}$-specific TCR, 318) ${ }^{13}$, RIPGP mice ${ }^{11}$, RIP-GP $\times$ RIP-BLC mice ${ }^{28}$, Rag $^{-1-}$ mice (Jackson Laboratory),

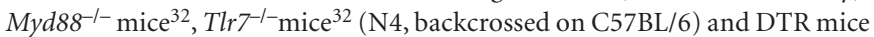
(for depletion of dendritic cells, DTR Jackson Laboratory) were maintained on the C57BL/6 genetic background, whereas Ifnagr ${ }^{-1-}$ mice $^{35}$ were of the $129 \mathrm{~Sv}$ genetic background. $T l r 3^{-1-}$ mice ${ }^{49}$ mice were on a BALB/c background. Ifnar ${ }^{-1-}$ and Ifnar ${ }^{+/-}$mice crossed on RIP-GP mice were of mixed background $(129 \mathrm{~Sv} \times \mathrm{C} 57 \mathrm{BL} / 6)$. For generation of bone marrow chimeras, recipient mice were irradiated with $950 \mathrm{rad}$ on day -1 . On day $0,10^{7}$ bone marrow cells were transferred and mice were used for experiments 7 weeks later. All mice were purchased from the Institute for Labortierkunde and housed under specific pathogen-free conditions. RIP-GP $\times$ RIP-BLC (and the corresponding RIP-GP mice) were used at an age of more than 10 weeks (to ensure the development of lymphoid structures within the pancreas of RIP-GP $\times$ RIP-BLC mice). Animal experiments including infection with recombinant viruses were carried out with authorization of the Veterinäramt of the Kanton Zurich and in accordance with the Swiss law for animal protection.

Immunization with peptides or Toll-like-receptor ligands, and adoptive transfer of CD8 $^{+}$T cells. gp33 (KAVYNFATM) was synthesized by Neosystems. Mice were immunized on day 0,2 and 5 with $1 \mathrm{mg}$ gp33 and $50 \mu \mathrm{g}$ CpG (ODN1826, Coley Pharmacia) and then boosted with peptide alone on day 9 . For priming of LCMV-gp33/H-2D ${ }^{\mathrm{b}}$-specific TCR-transgenic CD8 ${ }^{+} \mathrm{T}$ cells, $10^{7}$ splenocytes were adoptively transferred in RIP-GP mice on day -1 , and mice were immunized with $1 \mathrm{mg}$ gp33 on day 0 , day 4 and day 9 . As the precursor frequency exceeded $1 \%$ of total $\mathrm{CD}^{+} \mathrm{T}$ cells, no further costimulation with $\mathrm{CpG}$ was required for priming. To analyze the influence of TLRs, we treated mice with $200 \mu \mathrm{g}$ poly(I:C) (Sigma-Aldrich), a ligand for TLR3; $20 \mu \mathrm{g}$ R-848 (InvivoGen), a ligand for TLR7; $50 \mu \mathrm{g} \mathrm{CpG} \mathrm{(ODN} \mathrm{1826),} \mathrm{a} \mathrm{ligand} \mathrm{for} \mathrm{TLR9;} \mathrm{or} 50 \mu \mathrm{g}$ lipopolysaccharide (Sigma-Aldrich) a ligand for TLR4. Recombinant mouse IFN- $\alpha$ was obtained from Hycult biotechnology ( $10^{4}$ units/mouse). For in vivo depletion of IFN- $\alpha$, a rat monoclonal antibody was used (RMMA-1, Biomedical Laboratories, PBL).

FACS analysis. FACS analysis was performed as described previously ${ }^{50}$. Briefly, splenocytes or peripheral blood lymphocytes were stained using phycoerythrinlabeled gp33 MHC class I tetramer (gp33/H-2D ${ }^{\mathrm{b}}$ ) for $15 \mathrm{~min}$ at $37^{\circ} \mathrm{C}$, followed by staining with antibody specific for CD8-APC (BD PharMingen) for $30 \mathrm{~min}$ at $4{ }^{\circ} \mathrm{C}$. For determination of their activation status, lymphocytes were stained with antibodies specific for CD62L, CD44, CD122 and Ly6c+g (BD PharMingen) for $30 \mathrm{~min}$ at $4^{\circ} \mathrm{C}$.

Cytotoxicity assay. ${ }^{51} \mathrm{Cr}$ release assays were performed as previously described. ${ }^{50}$ EL4 target cells were loaded with ${ }^{51} \mathrm{Cr}$ and pulsed with or without gp33. Splenocytes of immunized mice were incubated directly ex vivo (Primary) or after restimulation with gp33 for $5 \mathrm{~d}$ (Secondary) with the target cells. Supernatant was assessed after $8 \mathrm{~h}$. We performed assays for in vivo cytotoxicity using C57BL/6 splenocytes incubated for $1 \mathrm{~h}$ with or without gp33 and labeled for $10 \mathrm{~min}$ with $5 \mu \mathrm{g} / \mathrm{ml}$ (peptide-labeled splenocytes) or $0.5 \mu \mathrm{g} / \mathrm{ml}$ (peptide nonlabeled splenocytes) carboxyfluorescein diacetate succinimidyl ester (CFSE, Molecular Probes), respectively. We injected $10^{7}$ cells of each fraction intravenously. The number of CFSE-positive cells remaining in the spleen after $4 \mathrm{~h}$ was determined by FACS analysis.

Blood glucose concentrations. Blood glucose concentrations were analyzed from a drop of blood using a Glucometer Elite (Bayer).

IFN- $\alpha$ ELISA. We performed IFN- $\alpha$ ELISA according to the manufacturers' specifications (Research Diagnostics RDI).

Histology. Histological analyses were performed on snap-frozen tissue. We stained sections with monoclonal antibodies against murine MHC I (M1/42) ${ }^{14}$, V 22 (Pharmingen), CD11b (BMA Biomedicals AG), CD11c (KB90, M0732; DAKO), B220 (RA3-6B2, 01121D; Pharmingen), or with VL4, a monoclonal antibody against the LCMV nucleoprotein ${ }^{48}$. Marginal zone was stained with MOMA-1 (BMA Biomedicals). For staining of IFN- $\alpha$, a polyclonal sheep antibody specific for mouse IFN- $\alpha$ antiserum was used (Biomedical Laboratories). Staining was developed using a goat anti-rat antibody (Caltag Laboratories) and an alkaline phosphatase-coupled donkey anti-goat antibody (Jackson ImmunoResearch) with naphthol AS-BI (6-bromo-2-hydroxy-3-naphtholic acid 2-methoxy anilide) phosphate and new fuchsin as a substrate. The presence of alkaline phosphatase activity yielded a red reaction product. The sections were counterstained with hemalum.

Note: Supplementary Information is available on the Nature Medicine website.

\section{ACKNOWLEDGMENTS}

We thank A. Aguzzi, M. Heikenwälder and C. Sigurdson for ideas, discussions and critical comments. Additionally, we thank K. Tschannen for technical support and A. Nowotny and S. Behnke for histological analysis. This study was supported by the Swiss National Science Foundation and Deutsche Forschungsgemeinschaft (DFG) LA1419/1-1. 


\section{COMPETING INTERESTS STATEMENT}

The authors declare that they have no competing financial interests.

Received 30 March; accepted 1 December 2004

Published online at http://www.nature.com/naturemedicine/

1. Roep, B.O. T-cell responses to autoantigens in IDDM. The search for the Holy Grail. Diabetes 45, 1147-1156 (1996).

2. Yang, Y. \& Santamaria, P. Dissecting autoimmune diabetes through genetic manipulation of non-obese diabetic mice. Diabetologia 46, 1447-1464 (2003).

3. Erlich, H.A. HLA class II sequences and genetic susceptibility to insulin dependent diabetes mellitus. Baillieres Clin. Endocrinol. Metab. 5, 395-411 (1991).

4. Trucco, M. \& Dorman, J.S. Immunogenetics of insulin-dependent diabetes mellitus in humans. Crit. Rev. Immunol. 9, 201-245 (1989).

5. Hyoty, H. \& Taylor, K.W. The role of viruses in human diabetes. Diabetologia 45, 13531361 (2002)

6. Roep, B.O. et al. Molecular mimicry in type 1 diabetes: immune cross-reactivity between islet autoantigen and human cytomegalovirus but not Coxsackie virus. Ann. NY Acad. Sci. 958, 163-165 (2002).

7. Schloot, N.C. et al. Molecular mimicry in type 1 diabetes mellitus revisited: T-cell clones to GAD65 peptides with sequence homology to Coxsackie or proinsulin peptides do not crossreact with homologous counterpart. Hum. Immunol. 62, 299-309 (2001).

8. Hiemstra, H.S. et al. Definition of natural T cell antigens with mimicry epitopes obtained from dedicated synthetic peptide libraries. J. Immunol. 161, 4078-4082 (1998).

9. Potena, L. et al. Hydroxymethyl-glutaryl coenzyme a reductase inhibition limits cytomegalovirus infection in human endothelial cells. Circulation 109, 532-536 (2004).

10. Roep, B.O. et al. T-cell reactivity to beta-cell membrane antigens associated with betacell destruction in IDDM. Diabetes 44, 278-283 (1995).

11. Ohashi, P.S. et al. Ablation of 'tolerance' and induction of diabetes by virus infection in viral antigen transgenic mice. Cell 65, 305-317 (1991).

12. von Herrath, M.G. \& Oldstone, M.B. Interferon-gamma is essential for destruction of beta cells and development of insulin-dependent diabetes mellitus. J. Exp. Med. 185 , 531-539 (1997)

13. Ludewig, B., Odermatt, B., Landmann, S., Hengartner, H. \& Zinkernagel, R.M. Dendritic cells induce autoimmune diabetes and maintain disease via de novo formation of local lymphoid tissue. J. Exp. Med. 188, 1493-1501 (1998)

14. Ohashi, P.S. et al. Induction of diabetes is influenced by the infectious virus and local expression of MHC class I and tumor necrosis factor-alpha. J. Immunol. 150, 5185-5194 (1993)

15. Nansen, A., Marker, O., Bartholdy, C. \& Thomsen, A.R. CCR2+ and CCR5+CD8 ${ }^{+}$T cells increase during viral infection and migrate to sites of infection. Eur. J. Immunol. 30, 1797-1806 (2000)

16. Frigerio, S. et al. Beta cells are responsible for CXCR3-mediated T-cell infiltration in insulitis. Nat. Med. 8, 1414-1420 (2002)

17. Hoshino, K., Kaisho, T., Iwabe, T., Takeuchi, O. \& Akira, S. Differential involvement of IFN- $\beta$ in Toll-like receptor-stimulated dendritic cell activation. Int. Immunol. 14, $1225-1231$ (2002)

18. Krug, A. et al. Toll-like receptor expression reveals CpG DNA as a unique microbial stimulus for plasmacytoid dendritic cells which synergizes with CD40 ligand to induce high amounts of IL-12. Eur. J. Immunol. 31, 3026-3037 (2001).

19. Kaisho, T. \& Akira, S. Regulation of dendritic cell function through Toll-like receptors. Curr. Mol. Med. 3, 759-771 (2003)

20. Dalod, M. et al. Interferon $\alpha / \beta$ and interleukin 12 responses to viral infections: pathways regulating dendritic cell cytokine expression in vivo. J. Exp. Med. 195, 517-528 (2002).

21. Doughty, L., Nguyen, K., Durbin, J. \& Biron, C. A role for IFN- $\alpha \beta$ in virus infectioninduced sensitization to endotoxin. J. Immunol. 166, 2658-2664 (2001).

22. Zhang, D. et al. A Toll-like receptor that prevents infection by uropathogenic bacteria. Science 303, 1522-1526 (2004)

23. Alexopoulou, L., Holt, A.C., Medzhitov, R. \& Flavell, R.A. Recognition of double-stranded RNA and activation of NF-KB by Toll-like receptor 3. Nature 413, 732-738 (2001).

24. Heil, F. et al. Species-specific recognition of single-stranded RNA via Toll-like receptor
7 and 8. Science 303, 1526-1529 (2004)

25. Hemmi, H. et al. A Toll-like receptor recognizes bacterial DNA. Nature 408, 740-745 (2000).

26. Poltorak, A. et al. Defective LPS signaling in $\mathrm{C} 3 \mathrm{H} / \mathrm{HeJ}$ and C57BL/10ScCr mice: mutations in TIr4 gene. Science 282, 2085-2088 (1998).

27. Meagher, C. et al. Cytokines and chemokines in the pathogenesis of murine type 1 diabetes. Adv. Exp. Med. Biol. 520, 133-158 (2003).

28. Luther, S.A., Lopez, T., Bai, W., Hanahan, D. \& Cyster, J.G. BLC expression in pancreatic islets causes $B$ cell recruitment and lymphotoxin-dependent lymphoid neogenesis. Immunity 12, 471-481 (2000).

29. Biron, C.A. Role of early cytokines, including alpha and beta interferons (IFN- $\alpha / \beta$ ), in innate and adaptive immune responses to viral infections. Semin. Immunol. 10 383-390 (1998).

30. Diebold, S.S., Kaisho, T., Hemmi, H., Akira, S. \& Reis, E.S.C. Innate antiviral responses by means of TLR7-mediated recognition of single-stranded RNA. Science 303, 15291531 (2004).

31. Diebold, S.S. et al. Viral infection switches non-plasmacytoid dendritic cells into high interferon producers. Nature 424, 324-328 (2003).

32. Hemmi, H. et al. Small anti-viral compounds activate immune cells via the TLR7 MyD88-dependent signaling pathway. Nat. Immunol. 3, 196-200 (2002).

33. Asselin-Paturel, C. et al. Mouse type I IFN-producing cells are immature APCs with plasmacytoid morphology. Nat. Immunol. 2, 1144-1150 (2001).

34. Ishikawa, R. \& Biron, C.A. IFN induction and associated changes in splenic leukocyte distribution. J. Immunol. 150, 3713-3727 (1993).

35. Ou, R., Zhou, S., Huang, L. \& Moskophidis, D. Critical role for $\alpha / \beta$ and $\gamma$ interferons in persistence of lymphocytic choriomeningitis virus by clonal exhaustion of cytotoxic $T$ cells. J. Virol .75, 8407-8423 (2001).

36. Malmgaard, L., Salazar-Mather, T.P., Lewis, C.A. \& Biron, C.A. Promotion of $\alpha / \beta$ interferon induction during in vivo viral infection through $\alpha / \beta$ interferon receptor/STAT1 system-dependent and -independent pathways. J. Virol. 76, 4520-4525 (2002).

37. Betterle, C. et al. Autoimmunity against pancreatic islets and other tissues before and after interferon-alpha therapy in patients with hepatitis $\mathrm{C}$ virus chronic infection. Diabetes Care 23, 1177-1181 (2000).

38. Serreze, D.V., Hamaguchi, K. \& Leiter, E.H. Immunostimulation circumvents diabetes in NOD/Lt mice. J. Autoimmun. 2, 759-776 (1989).

39. Sobel, D.O. et al. Poly I:C induces development of diabetes mellitus in BB rat. Diabetes 41, 515-520 (1992)

40. Ewel, C.H., Sobel, D.O., Zeligs, B.J. \& Bellanti, J.A. Poly I:C accelerates development of diabetes mellitus in diabetes-prone BB rat. Diabetes 41, 1016-1021 (1992).

41. Sobel, D.O. et al. Low dose poly I:C prevents diabetes in the diabetes prone BB rat. $J$. Autoimmun. 11, 343-352 (1998)

42. Langenkamp, A., Messi, M., Lanzavecchia, A. \& Sallusto, F. Kinetics of dendritic cell activation: impact on priming of $\mathrm{T}_{H} 1, \mathrm{~T}_{\mathrm{H}} 2$ and nonpolarized T cells. Nat. Immunol. 1 , 311-316 (2000).

43. Kobayashi, K. et al. IRAK-M is a negative regulator of Toll-like receptor signaling. Cell 110, 191-202 (2002)

44. Kawai, T., Adachi, O., Ogawa, T., Takeda, K. \& Akira, S. Unresponsiveness of MyD88deficient mice to endotoxin. Immunity 11, 115-122 (1999).

45. O'Neill, L.A. The interleukin-1 receptor/Toll-like receptor superfamily: signal transduction during inflammation and host defense. Sci. STKE 2000, RE1 (2000)

46. Akira, S. The role of IL-18 in innate immunity. Curr. Opin. Immunol. 12, 59-63 (2000).

47. Pien, G.C., Nguyen, K.B., Malmgaard, L., Satoskar, A.R. \& Biron, C.A. A unique mechanism for innate cytokine promotion of T cell responses to viral infections. J. Immunol. 169, 5827-5837 (2002)

48. Battegay, M. et al. Quantification of lymphocytic choriomeningitis virus with an immunological focus assay in 24- or 96-well plates. J. Virol. Methods 33, 191-198 (1991) Published errata appear in J. Virol. Methods 35, 115 (1991) and 38, 263 (1992).

49. Honda, K. et al. Selective contribution of IFN- $\alpha / \beta$ signaling to the maturation of dendritic cells induced by double-stranded RNA or viral infection. Proc. Natl. Acad. Sci. USA 100, 10872-10877 (2003).

50. Junt, T. et al. Antiviral immune responses in the absence of organized lymphoid T cell zones in plt/plt mice. J. Immunol. 168, 6032-6040 (2002). 